Original study

\title{
Breast cancer with intraabdominal metastases. Is surgery necessary?
}

\author{
Andrian Panuța ${ }^{1,2}$, Iulian Radu*, ${ }^{*, 2}$, Ionuț Huțanu ${ }^{1,2}$, Ana-Maria Mușina ${ }^{1,2}$, Mihaela \\ Buna-Arvinte $^{1,2}$, Mihaela Mădălina Gavrilescu ${ }^{1,2}$, Dragoș Viorel Scripcariu ${ }^{1,2}$, Viorel \\ Scripcariu $^{1,2}$
}

${ }^{1}$ Ist Surgical Oncology Unit, lasi Regional Cancer Institute, Romania; ${ }^{2}$ Department of Surgery, "Grigore T. Popa" University of Medicine and Pharmacy lasi, Romania

\begin{abstract}
Metastatic breast cancer has a very poor prognosis, considering the lack of reliable curative medical or surgical approaches. Patients with stage IV breast cancer usually undergo palliative surgical procedures and symptomatic treatment. In a 5-year period, 1258 patients with breast cancer were treated in our surgical oncology unit. For the current study, we have selected $19(1.43 \%)$ female patients with intraabdominal metastases (peritoneal, hepatic, ovarian etc.) derived from breast cancer, which received at least one surgical procedure in our unit. We compared our data with up-to-date reports and guidelines in order to establish the role and further directions of surgery, and (most importantly) the necessity of surgery itself in the management of this therapeutically disadvantaged patient-group. Even if current guidelines do not recommend surgical treatment of intraabdominal metastases derived from breast cancer, several oncology centers (including our unit) did not discard surgery, especially in patients with solitary metastasis.
\end{abstract}

Keywords: metastatic breast cancer, peritoneal metastases, liver metastases, ovarian metastases, unusual origin metastases, cytoreductive surgery, HIPEC

\section{Introduction}

Breast cancer $(\mathrm{BC})$ is the most frequent malignant tumor and the major cause of cancer-related death among women. If diagnosed in postmenopausal women, early stages (Tis-2N0-1M0) and with a favorable biology (luminal molecular subtypes) BC represents a perfect example of a curable cancer. Life expectancy for patients with locally advanced or recurrent non-metastatic $B C$ has also increased due to the

Received: May 2018; Accepted after review: November 2018; Published: December 2018.

*Corresponding author: Iulian Radu, Department of Surgery, Regional Oncology Institute, 35 Gral Berthelot str., lasi, Romania.

Email: raduiuli@gmail.com advancements of chemo-, hormonal-, radioand targeted therapies [1].

On the other hand, metastatic breast cancer (MBC) does not benefit from any curative procedures and therefore represents quite a challenge for breast surgeons and oncologists. Its incidence remained stable through the years even if screening programs showed their efficiency, and breast cancer is diagnosed, in the majority of cases, in the early stages (at least in Western Europe). Apparently, metastases do not always occur in locally advanced breast cancer. Another explanation may be the fact that MBC affects younger patients that are not included in breast cancer screening programs [2].

The role of surgery in stage IV breast cancer with intraabdominal metastases is highly debatable. Widely recognized, up to date guidelines for clinical practice (such as 
those published by the National Comprehensive Cancer Network (NCCN) [3] and the European Society for Medical Oncology (ESMO)) [4] do not recommend primary breast tumor surgery or excision of metastases in MBC. In addition, there are no randomized studies to assess the benefit of axillary lymph node dissection and usually a sentinel node biopsy is performed for staging purposes. Nevertheless, several studies affirm the possible benefit of breast surgery (lumpectomy or mastectomy), this can be explained by the reduction of the tumor burden, decreasing the level of circulating tumor cells. This fact has a potential influence on the prognosis of metastatic breast cancer. However, in the majority of cases breast surgery, in such situations, is performed only if the tumor is symptomatic (ulcerated, infected, hemorrhagic breast lump) [3-5].

Besides breast surgery, as a general surgery team, we perform a wide range of operations, mostly in the abdominal cavity, for primary and secondary digestive, urologic and genital cancers, which brings intraabdominal metastases from breast cancer in our sphere of interest. Our objective was to share the experience of our unit in surgical treatment of metastatic breast cancer in order to compare our results with those shown by reference surgical oncology centers.

\section{Patients and Methods}

In this study, we retrospectively analyzed cases of $\mathrm{BC}$, who received surgical treatment in our unit (First Surgical Oncology Unit, Regional Institute of Cancer lasi, Romania). Over the course of 5 years (May 2012 - May 2017), a number of 1258 female patients were operated in our unit for breast cancer. Nineteen cases $(1.43 \%)$ of MBC were included in or study. These patients showed peritoneal carcinomatosis, liver, ovarian, adrenal, and uterine metastases (with or without extraabdominal metastases). We have included MBC patients with one or more metastases located within the abdominal cavity, who were hospitalized in our unit and underwent at least one surgical procedure.

Clinicopathological characteristics of the patients: The studied group contained 19 female patients with an average age of 63 ranging from 46 to 76 years old. Urban/rural living environment was attested in equal proportions. All patients received physical examination, mammary ultrasound and mammography, abdominal and pelvic ultrasound (with additional CT or MRI scans where needed) and were histologically diagnosed preoperatively (core-biopsy) or intraoperatively (extemporaneous exam). Most patients were diagnosed with locally advanced breast cancer (T3-T4). Twelve cases (1 case associated with adrenal metastasis) were diagnosed with liver metastases (Figure 1) with or without extra-abdominal masses (lungs, bones, brain) and 7 cases with peritoneal carcinomatosis (Figures 2 and $3 a$ ) with or without ovarian secondary dissemination (Figure 3b), and 1 case of ovarian metastasis (without carcinomatosis). Eleven cases were considered as PMBC (primary metastatic breast cancer). In 8 cases, metastatic disease occurred at an important time-span from breast surgery (6 months - 4 years). The predominant histological type (15 cases) was NST (no special type/invasive ductal carcinoma), while 4 cases were of invasive lobular carcinoma. Molecular subtypes found in the study group: luminal B 10 cases, luminal $A-6$ cases, triple negative -2 cases and HER2-type - 1 case.

Treatment. All cases received pre- or postoperative chemotherapy \pm antiestrogen therapy and, in locally advanced stages, palliative external radiotherapy. 


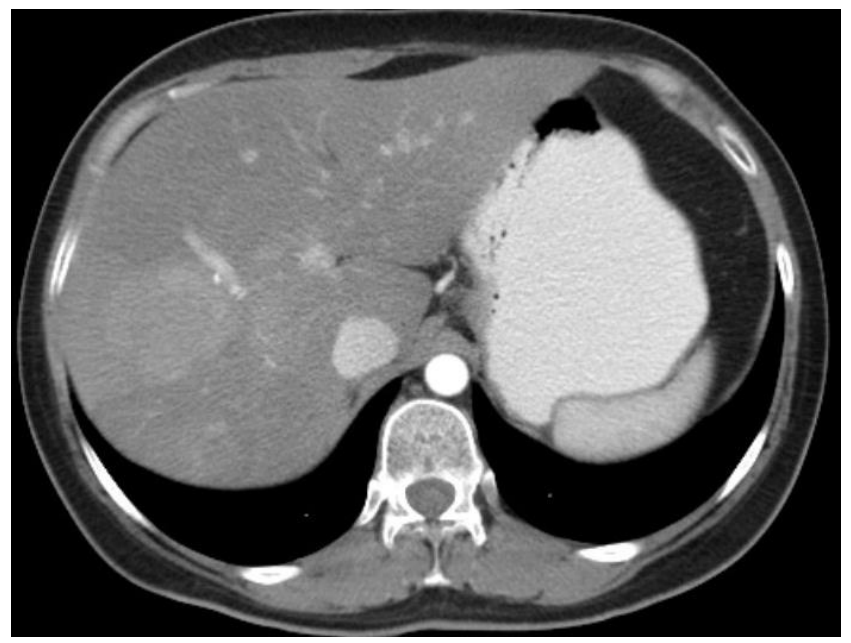

Fig. 1. Liver metastases from breast cancer

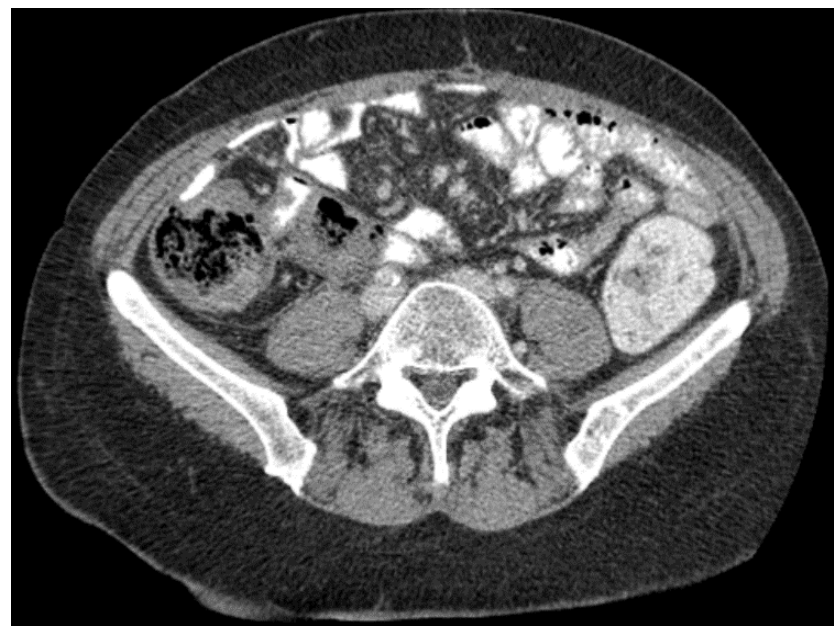

Fig. 2. Peritoneal carcinomatosis from breast cancer

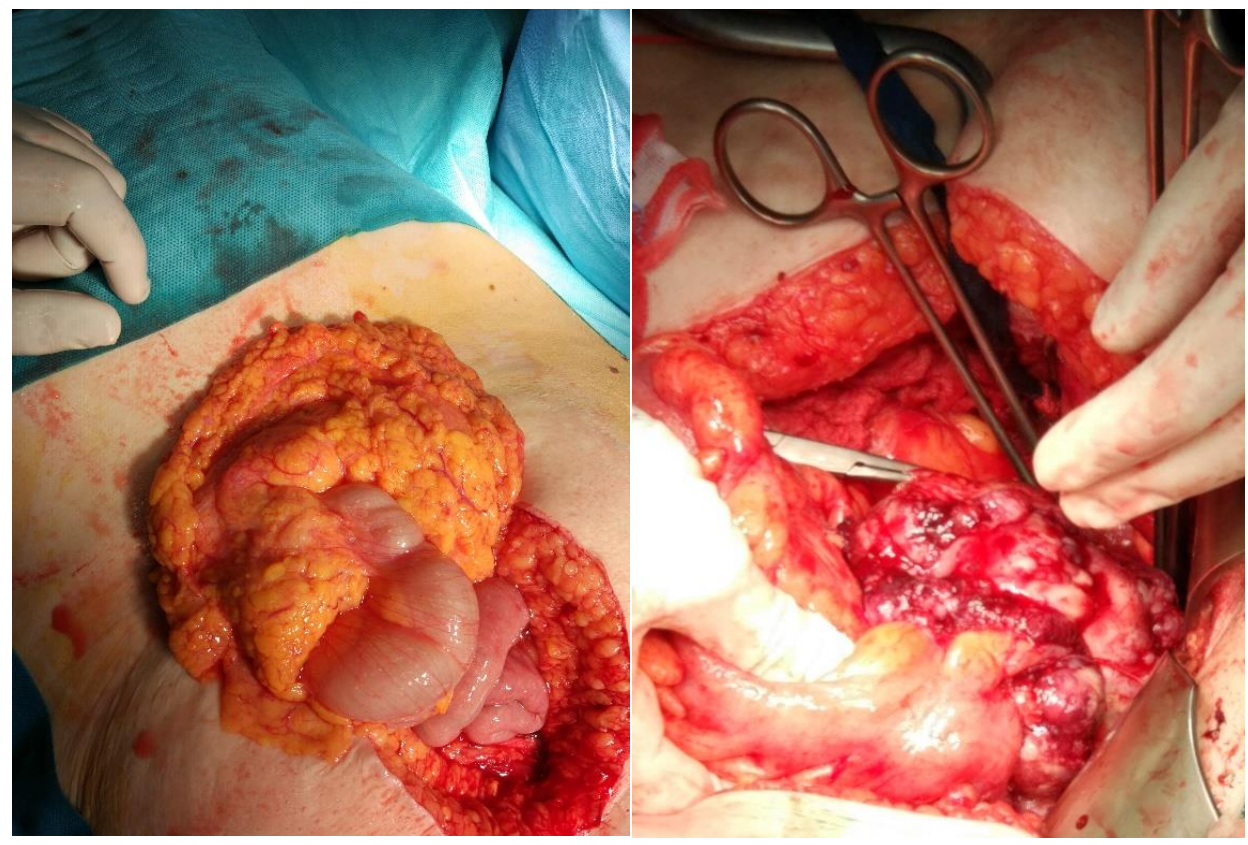

Fig. 3. Peritoneal carcinomatosis (a) and ovarian metastasis (b) derived from breast cancer 
Surgical treatment applied on our BC patients with intraabdominal metastases (Table 1) had mainly a palliative intent. Only one patient had a solitary hepatic metastasis which was feasible for R0 resection. In seven cases, breast cancer surgery was performed 6 months to 4 years prior the occurrence of metastatic disease. Another patient with a solitary ovarian metastasis (without PC) underwent a hysterectomy with bilateral anexectomy with curative intent. In 10 cases, palliative surgery of primary breast tumor was performed.

Table 1. Surgical procedures performed in our unit for patients Stage IV breast cancer with intraabdominal metastases

\begin{tabular}{lcc} 
Surgery performed on the primary breast tumor & No. & $\%$ \\
Subcutaneous mastectomy & 1 & 5.26 \\
\hline Total/,cleansing” mastectomy & 4 & 21.05 \\
\hline Bilateral total mastectomy & 1 & 5.26 \\
\hline Madden's modified radical mastectomy & 2 & 10.52 \\
\hline Lumpectomy & 2 & 10.52 \\
\hline Excision of the recurrent prepectoral tumor & 1 & 5.26 \\
\hline Core-biopsy (alone) & 2 & 10.52 \\
No breast surgery (primary tumor being operated before the admission to our unit) & 6 & 31.56 \\
\hline Surgery performed on the metastases from breast tumor & No. & $\%$ \\
\hline Hepatic metastasectomy (partial IV segmentectomy) & 1 & 5.26 \\
\hline Subtotal hysterectomy with bilateral anexectomy & 2 & 10.52 \\
\hline Unilateral anexectomy & 1 & 5.26 \\
\hline Laparoscopic peritoneal biopsy & 1 & 5.26 \\
\hline Terminal colostomy (for occlusive syndrome caused by PC) & 1 & 5.26 \\
\hline von Hacker's gastroenterostomy (for upper gastrointestinal obstruction syndrome & 1 & 5.26 \\
caused by PC) and multiple peritoneal biopsies & 11 & 57.89 \\
\hline No surgery on metastases & 1
\end{tabular}

Follow-up: Our patients were followed-up every 3 months in first 2 years and every 6 months during the third-fifth years after surgery, by physical examination (both by a surgeon and an oncologist), tumor marker CA15.3 determination and bilateral breast and abdominal ultrasound. Also in some cases mammography, thoracic-abdominal CT and bone scintigraphy were performed.

Outcomes: Only 7 out of 19 patients in the study group survived (overall survival $36.8 \%$ ).

From our patients' database, we would mention some cases that do not fit the disease evolution patterns.

Case 1. A 55-years-old female patient was admitted to our unit for performing a cytoreductive surgery for a high suspicion of stage IIIC ovarian carcinoma (left ovarian tumor, an important amount of ascites, multiple nodules of peritoneal carcinomatosis revealed on CT scan, increased level of CA125 tumor marker (677 units $/ \mathrm{mL}))$. The patient underwent a subtotal hysterectomy with bilateral anexectomy. The pathological result was surprising - Microscopy: ovarian and tubal fragments (containing many mesonephric debris) with diffuse carcinoma infiltration with trabecular and cordial architecture and discoesic alveolus, reduced associated stroma and major edema. Immunohistochemistry (IHC): EMA, CK7, ER, PR, MUC5AC, GCDFP15, Mammaglobin, CK20 - positive in tumor cells. TTF1, CDX2 negative in tumor cells. E-cadherin - positive in tumor cells. HER2neu - negative. Ki67 positive in about $20-22 \%$ of tumor cells. Diagnosis: The histological and IHC aspects described advocate for a carcinomatous infiltration within a carcinoma metastasis with a mammary gland origin. Further investigations revealed bilateral multicentric breast cancer. After the patient underwent 6 cycles of chemotherapy (Cyclophosphamide $1000 \mathrm{mg}+$ Epirubicin 150mg) combined with hormonal treatment (Tamoxifen), a bilateral total mastectomy was performed (Pathology 
result: NST invasive carcinoma pT3mNxG1 (left breast), pT2mNxG1 (right breast)). We regularly followed this patient for more than 4 years. She presents no signs of local recurrence or systemic disease progression.

Case 2. A 52-years-old female patient known with radically operated, chemo-treated right breast cancer (in another institution) was admitted (18 months after the initial diagnosis of $\mathrm{BC}$ ) in our unit in order to evaluate a hepatic tumor mass revealed on a CT-scan (solitary $16 / 17 / 19 \mathrm{~mm}$-sized hepatic tumor located in the fifth hepatic segment), performed because of an increased level of CA15.3 tumor marker (55.82 units $/ \mathrm{mL}$ ). The patient underwent surgery; intraoperatively, the location of the liver mass proved to be in the fourth posterior segment - a partial hepatic segmentectomy was performed. The pathology examination confirmed the breast cancer origin of the hepatic metastasis (microscopy: a hepatic fragment exhibiting adenocarcinoma metastasis with tubular architecture and reduced stroma. IHC: CK7 - positive in less than $20 \%$ of tumor cells. Mammaglobin, GCDFP15 - positive in tumor cells. ER, PR diffusely and intensely positive in tumor cells. Ki67 - positive in about 15\% of tumor cells. HER2neu - negative in tumor cells. CK20 negative in tumor cells. Conclusion: The described aspects advocate for the diagnosis of metastasis with a mammary gland origin). We regularly followed this patient for more than 2 years. She presents no signs of local recurrence or systemic disease progression.

Case 3. A 47-years-old female patient known with radically operated, bilateral metachronous breast cancer (with bilateral breast reconstruction in another institution), under continuous antiestrogen therapy, was admitted to our unit in order to evaluate the cause of a recently appeared menometrorrhagia. Abdominal CT and MRI scans revealed multiple bone metastases, an ovarian tumor and a tumorous mass in the cervix. The patient underwent unilateral oophorectomy and cervical biopsy. Both of these organs proved to be metastatic sites for breast carcinoma. The patient's evolution was unfavorable. The particularity of this case is the very rare site of metastases from breast cancer, namely the cervix.

\section{Discussions}

Nowadays, breast cancer surgery has evolved, offering high rates of long-term survival and an acceptable quality of life (breast conserving surgery, immediate breast reconstruction etc.) for BC patients. Unfortunately, metastatic breast cancer (MBC) remains a life-threatening condition with no appropriate therapeutic solutions. During their lifetime, 20 to $40 \%$ of BC patients will develop metastatic disease [2,6]. Up to $10 \%$ of stage IV breast cancers prove to be primary metastatic - metastases being diagnosed simultaneously with primary breast tumor [2]. The dissemination of metastases is frequently multiorganic. Preferred dissemination sites include bones (67\%), lungs (31\%), liver (35\%), brain $(11 \%)$, skin $(7.5 \%)$ and other sites (peritoneum $(7.6 \%)$, ovary $(1.5 \%)$, etc.) $[1,2]$.

Locoregional treatment of MBC. NCCN and ESMO guidelines do not recommend any kind of surgery of primary or secondary tumors with curative intent, since there are no randomized studies to demonstrate its survival benefits in comparison to chemotherapy alone [3-6]. The only recommended surgery in MBC is bilateral oophorectomy in luminal molecular subtypes (ER/PR positive) in premenopausal women and palliative ("cleansing") total mastectomy in case of locally advanced, ulcerated, infected or hemorrhagic breast cancer $[4,5]$. We should mention that in the majority of $\mathrm{BC}$ patients diagnosed with metastatic disease, primary breast tumor and axillary surgery have already been performed, detectable metastases usually occurring several months or years after diagnosis. In case of PMBC, the role of surgery of primary breast tumor is minor. In non-locally advanced cases a core-biopsy is usually the only surgical procedure needed, the patient only receiving chemotherapy alone [7].

The role of surgery in hepatic metastases derived from BC. Although the liver is the most common intraabdominal site of breast cancer metastases (hepatic metastases found in up to $75 \%$ of patients deceased due to $\mathrm{BC}$ ) the frequency of solitary liver metastasis is low (3$9 \%)$. Traditionally chemotherapy and surgical treatment of MBC cases have a palliative intent only. There are no randomized studies 
demonstrating the advantages of the excision of intraabdominal metastases derived from BC in comparison with chemotherapy alone. However, MBC patients with single liver metastases can become candidates for metastasectomy [8]. There are several publications attesting an increase in survival among patients with isolated hepatic metastases from BC who underwent hepatic resections. The survival benefit of this procedure mainly depends on the dimensions of the hepatic tumor $(<5 \mathrm{~cm}$ in diameter $)$, on the molecular biology of BC (better outcomes in hormone responsive molecular subtypes) and on the menopausal status of the patients (better results for patients older than 50 years). The response to neoadjuvant chemoand/or hormonal therapy is also an important selection criterion for eventual hepatic metastasectomy. Patients with primary metastatic diseases have poorer prognosis and benefit less from hepatectomy than those in whom liver metastasis occur more than one year after breast cancer diagnosis [5, 8, 9]. Despite related criteria of selection of the candidates for hepatectomy in MBC, a few single institution studies report favorable initial results in patients with more than one liver metastases, and more than that, even perform re-resection of hepatic metastases derived from $B C[10,11]$.

The role of surgery in peritoneal carcinomatosis derived from BC. Peritoneal carcinomatosis $(P C)$ is a diagnosis with a very poor prognosis and usually these patients receive palliative chemotherapy and symptomatic care [12]. PC usually occurs in patients with cancers of the digestive tract or of the female genital system. Recent papers evoked lobular invasive histologic type, high tumor grade, locally advanced cases as risk factors for breast cancer peritoneal metastases [13]. Survival rates in this group of patients are comparable to BC patients with brain metastases [10]. In specialized surgical oncology centers (including our unit) accurately selected patients with primary peritoneal malignant tumors (i.e. peritoneal mesothelioma and peritoneal pseudomyxoma peritonei) and PC derived from colon or ovarian cancers receive optimal cytoreductive surgery accompanied by hyperthermic intraperitoneal chemotherapy (HIPEC) with a curative intent with encouraging outcomes. Current guidelines show no indications for this procedure in MBC patients with peritoneal secondary determinations, but there are ongoing studies that applied HIPEC in limited series of patients with promising initial results $[14,15]$.

\section{Conclusions}

Intraabdominal metastatic disease rarely occurs in the progression of breast cancer and is usually associated with a locally advanced breast tumor (in our study, especially liver metastases associated with more frequent sites: lungs, bones, brain). Peritoneal carcinomatosis, ovarian metastases more often occur over a time span (on average 2 years in our study) from breast surgery. The lobular type of breast carcinoma tropism for serous membranes is evoked in literature and we obtained similar results (4 of 6 cases with PC revealed a lobular invasive breast carcinoma origin). Routinely used - abdominal ultrasound can identify eventual liver metastases, but has a poor specificity in detecting early peritoneal metastases. An abdominal CT scan for all patients diagnosed with breast cancer would certainly increase the management costs, but would also significantly contribute to the detection of primary metastatic breast cancer and to early diagnosis of intra-abdominal metastases from breast cancer. The majority of stage IV breast cancer patients treated in our unit were cases of primary metastatic breast cancer, which has more unfavorable outcomes than cases with metachronous metastases. This fact explains very poor survival rates in the studied group. The role of surgery in metastatic breast cancer is controversial and currently, in the majority of cases, it has either palliative intent or has no benefits at all. The combination of targeted therapy and cytoreductive surgery as well as the use of HIPEC in the treatment of metastatic breast cancer (with peritoneal carcinomatosis) is the subject of ongoing clinical trials. 


\section{Consent}

Consent Written informed consent was obtained from all the patients whose clinical data was used for the publication of this study.

\section{References}

1. Khanfir A, Lahiani F, Bouzguenda R, Ayedi I, Daoud J, Frikha M. Prognostic factors and survival in metastatic breast cancer: a single institution experience. Rep Pract Oncol Radiother 2013; 18(3):127-132.

2. Bertozzi S, Londero A, Cedolini C, Uzzau A, Seriau L, Bernardi S, et al. Prevalence, risk factors, and prognosis of peritoneal metastasis from breast cancer. SpringerPlus 2015; 4(1).

3. National Comprehensive Cancer Network. NCCN Guidelines Insights: Breast Cancer Version 1.2017.

4. Senkus E, Kyriakides S, Ohno S, PenaultLlorca F, Poortmans P, Rutgers E, et al. Primary breast cancer: ESMO Clinical Practice Guidelines for diagnosis, treatment and followup. Ann Oncol 2015; 26(suppl 5):v8-v30.

5. Ruiterkamp J, Ernst M. The role of surgery in metastatic breast cancer. Eur J Cancer 2011; 47:S6-S22.

6. Bland KI, Klimberg VS, Copeland EM, Gradishar WJ. The breast: comprehensive management of benign and malignant diseases. Philadelphia, PA: Elsevier; 2018.

7. Barinoff $J$, Schmidt $M$, Schneeweiss $A$, Schoenegg $W$, Thill M, Keitel $S$ et al. Primary metastatic breast cancer in the era of targeted therapy - Prognostic impact and the role of breast tumour surgery. Eur J Cancer 2017; 83:116-124.

8. Honoré C, Goéré D, Macovei R, Colace L, Benhaim L, Elias D. Peritoneal carcinomatosis from unusual cancer origins: Is there a role for hyperthermic intraperitoneal chemotherapy? J Visc Surg 2016; 153(2):101-107.

\section{Competing interests}

The authors declare that they have no competing interests.
9. Abbas H, Erridge S, Sodergren M, Papoulas M, Nawaz A, Menon $\mathrm{K}$ et al. Breast cancer liver metastases in a UK tertiary centre: Outcomes following referral to tumour board meeting. Int $J$ Surg 2017; 44:152-159.

10. Mariani $P$, Servois $V$, De Rycke $Y$, Bennett $S$, Feron J, Almubarak $M$ et al. Liver metastases from breast cancer: Surgical resection or not? A case-matched control study in highly selected patients. Eur J Surg Oncol 2013; 39(12):1377-1383.

11. Bacalbasa N, Balescu I, Dima S, Brasoveanu V, Popescu I. Does re-resection of liver metastases for breast cancer hepatic metastases improve survival? HPB 2016; 18:e187.

12. Garg R, Zahurak M, Trimble E, Armstrong D, Bristow R. Abdominal carcinomatosis in women with a history of breast cancer. Gyn Oncol 2005; 99(1):65-70.

13. Brinkman D, Misra $S$, Aydin N. A case report of symptomatic gallbladder disease in the setting of peritoneal carcinomatosis originating from invasive lobular carcinoma of the breast. Int $\mathrm{J}$ Surg Case Rep 2016; 24:60-62.

14. Cardi M, Sammartino $P$, Mingarelli V, Sibio $S$, Accarpio F, Biacchi D et al. Cytoreduction and HIPEC in the treatment of "unconventional" secondary peritoneal carcinomatosis. World $J$ Surgical Oncol 2015; 13(1):305-311.

15. Cardi $M$, Sammartino $P$, Framarino $M$, Biacchi D, Cortesi E, Sibio $S$ et al. Treatment of peritoneal carcinomatosis from breast cancer by maximal cytoreduction and HIPEC: A preliminary report on 5 cases. Breast 2013; 22(5):845-849. 